\title{
Erratum to: Mortality and treatment costs of hospitalized chronic kidney disease patients between the three major health insurance schemes in Thailand
}

Sirirat Anutrakulchai ${ }^{1 *}$, Pisaln Mairiang ${ }^{1}$, Cholatip Pongskul ${ }^{1}$, Kaewjai Thepsuthammarat ${ }^{2}$, Chitranon Chan-on ${ }^{1}$ and Bandit Thinkhamrop ${ }^{3 *}$

\section{Erratum}

Upon publication of this article [1], it was brought to our attention that Table 1 contained several errors. The correct Table 1 is shown below. This has been updated in the original article.

\begin{abstract}
Author details
${ }^{1}$ Department of Medicine, Faculty of Medicine, Khon Kaen University, Khon Kaen, Khon Kaen Province 40002, Thailand. ${ }^{2}$ Clinical Epidemiology Unit, Faculty of Medicine, Khon Kaen University, Khon Kaen, Khon Kaen Province 40002, Thailand. ${ }^{3}$ Department of Biostatistics and Demography, Faculty of Public Health, Khon Kaen University, Khon Kaen, Khon Kaen Province 40002, Thailand.
\end{abstract}

Received: 21 October 2016 Accepted: 21 October 2016

Published online: 25 October 2016

\section{Reference}

1. Anutrakulchai S, et al. Mortality and treatment costs of hospitalized chronic kidney disease patients between the three major health insurance schemes in Thailand. BMC Health Serv Res. 2016;16:528. doi:10.1186/s12913-016-1792-9.

\footnotetext{
* Correspondence: sirirt_a@kku.ac.th; bandit@kku.ac.th

${ }^{1}$ Department of Medicine, Faculty of Medicine, Khon Kaen University, Khon Kaen, Khon Kaen Province 40002, Thailand

${ }^{3}$ Department of Biostatistics and Demography, Faculty of Public Health, Khon

Kaen University, Khon Kaen, Khon Kaen Province 40002, Thailand

Full list of author information is available at the end of the article
} 
Table 1 Characteristics of CKD patients by the main three health schemes

\begin{tabular}{|c|c|c|c|}
\hline \multirow[t]{2}{*}{ Characteristics } & \multicolumn{3}{|c|}{ The main three Thai health schemes } \\
\hline & Universal Coverage Scheme & $\begin{array}{l}\text { Civil Servant Medical } \\
\text { Benefit Scheme }\end{array}$ & $\begin{array}{l}\text { Social Health } \\
\text { Insurance }\end{array}$ \\
\hline Number of adult patients (persons) & 98,727 & 24,767 & 4,844 \\
\hline Number of admissions (times) & 185,161 & 42,348 & 8,930 \\
\hline Age $($ mean $\pm S D)$ & $66.55 \pm 13.36$ & $72.23 \pm 11.46$ & $46.99 \pm 12.16$ \\
\hline Sex (male/female) & $1 / 1.15$ & $1 / 0.88$ & $1 / 0.58$ \\
\hline \multicolumn{4}{|l|}{ Region (\%) } \\
\hline$N / N E / C / S$ & 21.4 / 46.2 / 24.2 / 8.2 & 17.4 / 32.6 / $39.5 / 10.5$ & $11.6 / 11.2 / 71.2 / 6.1$ \\
\hline \multicolumn{4}{|l|}{ Hospital levels (\%) } \\
\hline Community hospital & 49.95 & 27.24 & 1.84 \\
\hline General hospital & 23.58 & 24.49 & 16.72 \\
\hline Tertiary hospital & 22.92 & 48.14 & 35.61 \\
\hline Private hospital & 3.55 & 0.13 & 45.83 \\
\hline Onetime admission / Multiple admission (\%) & $62.6 / 37.4$ & $64.8 / 35.2$ & $60.0 / 40.0$ \\
\hline CKD diagnosed as primary / secondary (\%) & $24.4 / 75.6$ & $19.9 / 80.1$ & $35.1 / 64.9$ \\
\hline Proportion of ESRD (\%) & 24.19 & 31.63 & 52.06 \\
\hline \multicolumn{4}{|l|}{ Common co-morbidities (\%) } \\
\hline Hypertension & 56.91 & 67.90 & 63.79 \\
\hline Diabetes mellitus & 45.08 & 49.09 & 36.50 \\
\hline Hyperlipidemia & 17.19 & 25.05 & 20.05 \\
\hline Ischemic heart disease & 13.49 & 21.68 & 11.91 \\
\hline Heart failure & 14.28 & 13.95 & 12.70 \\
\hline Gout & 10.49 & 13.17 & 6.44 \\
\hline Sepsis & 12.78 & 14.71 & 10.90 \\
\hline Pneumonia & 9.94 & 12.25 & 8.20 \\
\hline Acute kidney injury & 8.59 & 10.26 & 7.23 \\
\hline Diarrhea & 8.63 & 8.34 & 7.51 \\
\hline Stroke & 6.51 & 11.41 & 5.66 \\
\hline Respiratory failure & 8.53 & 7.85 & 4.81 \\
\hline \multicolumn{4}{|l|}{ Complications (\%) } \\
\hline Anemia requiring blood transfusion & 31.86 & 23.24 & 31.32 \\
\hline Hyperkalemia & 15.95 & 11.56 & 11.50 \\
\hline Volume overload & 12.44 & 8.96 & 14.80 \\
\hline Metabolic acidosis & 9.20 & 5.04 & 4.81 \\
\hline \multicolumn{4}{|l|}{ Dialysis treatment (\% of admissions) } \\
\hline Hemodialysis & 6.97 & 16.64 & 24.15 \\
\hline Peritoneal dialysis & 2.98 & 1.86 & 2.15 \\
\hline Overall mortality rate (\%) & 10.39 & 12.44 & 8.71 \\
\hline \multicolumn{4}{|l|}{ Mortality rate in different hospital levels (\%) } \\
\hline Community / General / Tertiary / Private & $3.7 / 14.9$ / 17.5 / 13.2 & 5.9 / 14.5 / $15.1 / 15.2$ & 7.9 / 8.04 / 10.4 / 7.6 \\
\hline
\end{tabular}

